\title{
Angleterre : La « révolution éducative » des academies
}

Donald Lillistone

\section{OpenEdition}

1 Journals

Édition électronique

URL : http://journals.openedition.org/ries/2439

DOI : $10.4000 /$ ries.2439

ISSN : 2261-4265

Éditeur

Centre international d'études pédagogiques

\section{Édition imprimée}

Date de publication : 1 septembre 2012

Pagination : 15-18

ISBN : 978-2-85420-595-4

ISSN : $1254-4590$

\section{Référence électronique}

Donald Lillistone, "Angleterre : La « révolution éducative » des academies », Revue internationale d'éducation de Sèvres [En ligne], 60 | septembre 2012, mis en ligne le 06 février 2015, consulté le 14 novembre 2019. URL : http://journals.openedition.org/ries/2439; DOI : 10.4000/ries.2439

Ce document a été généré automatiquement le 14 novembre 2019.

(C) Tous droits réservés 


\title{
Angleterre : La « révolution éducative » des academies
}

\author{
Donald Lillistone
}

Avant la formation en mai 2010 du gouvernement de coalition, il existait déjà, dans le système éducatif anglais ${ }^{1}$, une multiplicité d'établissements scolaires.

- La loi de 1988 avait augmenté de manière significative l'autonomie de tous les établissements financés par l'État, mais le niveau de cette autonomie varie selon le statut légal de chaque type d'établissement.

- Les city technology colleges (loi de 1988) et les academies (initiative de 2000) sont financés directement par le gouvernement central. Ce sont des établissements sponsorisés par des mécènes, et ils sont indépendants des autorités locales (Local Authorities: LA). Ils déterminent les conditions de service, les barèmes de salaire, et les inscriptions des élèves. Ces établissements ne sont pas tenus de suivre les programmes nationaux.

2 Il existe quatre catégories de maintained schools. Ces établissements sont financés par l'intermédiaire des LA, qui retiennent un pourcentage (environ $10 \%$ du budget de chaque établissement) pour financer leurs services. Les conditions de service et les barèmes de salaire sont déterminés au niveau national pour tous ces établissements, qui sont tenus de suivre les programmes nationaux.

3 Les établissements publics (community schools) et les établissements confessionnels (voluntary aided schools) forment la base de la comprehensive school ou collège unique (les établissements confessionnels en Angleterre sont gratuits²). Les autorités locales déterminent les inscriptions des établissements publics, tandis que les conseils d'administration déterminent celles des établissements confessionnels, ce qui est aussi le cas pour les foundation schools (loi de 1998).

- Les grammar schools sont des établissements sélectifs. Ces établissements existent toujours dans certaines LA malgré la loi de 1976, dont le but était de mettre fin à la sélection.

4 Tous les établissements sont tenus de respecter le School Admissions Code (code national des inscriptions). 
Pourquoi cette multiplicité d'établissements scolaires ? C'est la loi de 1980 adoptée par le gouvernement de Margaret Thatcher qui a proposé aux parents le choix de l'école, et c'est la loi de 1988 qui a ouvert la porte à la création de nouveaux types d'écoles secondaires. Une décennie plus tard, le gouvernement de Tony Blair a adopté le slogan « choix et diversité » pour toutes ses initiatives dans le domaine des services publics, et a continué à accroître la diversité des établissements secondaires.

6 Strictement parlant, les parents ne choisissent pas l'établissement dans lequel leurs enfants seront scolarisés. La loi leur donne plutôt le "droit d'exprimer une préférence ", et c'est ensuite aux LA et aux établissements de travailler ensemble pour répondre aux demandes des parents dans la mesure du possible. En septembre 2011, $84,6 \%$ des parents ont vu leur premier vœu d'établissement secondaire satisfait, mais avec de très grandes différences selon les régions (à Londres, ce chiffre n'était que de $66,2 \%)$.

7 Si deux gouvernements successifs, le premier conservateur, le second travailliste, partageaient la conviction que le résultat de "choix et diversité» serait une amélioration du système éducatif dans son ensemble, ce n'était pas l'avis de tout le monde. D'après Ron Glatter, professeur émérite d'administration de l'éducation de l' Open University, il faut « regarder au-delà de notre obsession pour les structures (...), au cours des deux dernières décennies, nous avons créé une multiplicité d'établissements différents, et cela n'a fait aucune différence (...). Les dangers sont évidents. Certains établissements - actuellement les academies en particulier - deviennent les favoris politiques dont il faut assurer le succès. $»^{3}$

8 Néanmoins, le nouveau gouvernement de coalition formé en mai 2010 par le conservateur David Cameron avec les libéraux-démocrates a aussitôt lancé ce que la presse anglaise appelle une " révolution éducative », en invitant tous les établissements scolaires (primaires et secondaires) à se convertir en academies sans nécessairement être sponsorisés par des mécènes. Initiative fort controversée et à laquelle les trois syndicats d'enseignants se sont opposés. Cependant, l'initiative a été accueillie avec enthousiasme par un nombre croissant d'établissements.

\section{La stratégie éducative du gouvernement}

9 La loi de 2010 permet la création de free schools, et les premières "écoles libres " ont ouvert leurs portes en septembre 2011. Ces établissements bénéficient du même statut légal que les academies. Une academy est un établissement public qui s'est converti, tandis qu'une free school est un tout nouvel établissement.

10 Le tableau ci-dessous permet de mesurer la rapidité avec laquelle le système éducatif est en train de se transformer, d'autant plus que les LA ne sont plus autorisées à créer de nouvelles écoles sous leur tutelle. Tout nouvel établissement est désormais soit une academy soit une free school. 


\begin{tabular}{|c|c|}
\hline \multicolumn{2}{|c|}{$\begin{array}{l}\text { Établissements secondaires financés } \\
\text { par l'État }\end{array}$} \\
\hline \multicolumn{2}{|c|}{ Septembre 2010} \\
\hline Community & 1706 \\
\hline Foundation & 779 \\
\hline Voluntary aided & 642 \\
\hline Academies & 202 \\
\hline Grammar schools & 164 \\
\hline City technology colleges & $3^{a}$ \\
\hline \multicolumn{2}{|c|}{ Septembre 2011} \\
\hline Community & 1157 \\
\hline Academies & $1050^{\mathrm{b}}$ \\
\hline Voluntary aided & 513 \\
\hline Foundation & 503 \\
\hline Grammar schools & 164 \\
\hline City technology colleges & 3 \\
\hline Free schools & 4 \\
\hline
\end{tabular}

a. 15 city technology colleges ont été créés entre 1988 et 1992 , dont 12 se sont convertis en academies avant 2010.

b. Dont 319 sponsorisées par des mécènes.

11 Le nombre d'établissements demandant à changer de statut ne cesse d'augmenter et, dans la mesure où les établissements peuvent changer de statut légal pendant l'année scolaire, le gouvernement a annoncé le $1^{\mathrm{er}}$ avril 2012 qu'il existait déjà 1776 academies (primaire et secondaire confondus), si bien que la plupart des établissements secondaires sont désormais hors du contrôle des autorités locales.

Quelle est la philosophie derrière cette "révolution éducative »? "Nous connaissons les dégâts provoqués par des services contrôlés de façon centralisée (...) La poigne de l'État doit être relâchée et le pouvoir confié aux gens (...) Le principe le plus important est celui de la diversité (...) Un autre principe clé est celui du choix. » C'est ainsi que le premier ministre, David Cameron, s'est exprimé en février 2011 dans un article du Daily Telegraph. Cette déclaration révèle le fil conducteur qui le relie directement à Margaret Thatcher ainsi qu'à Tony Blair.

Selon le gouvernement, les avantages des academies sont les suivants :

- indépendance par rapport aux LA (la « poigne » de l'État);

- autonomie pour déterminer les programmes scolaires;

- autonomie pour déterminer les conditions de service (y compris le nombre de jours travaillés par année scolaire et la durée de la journée de l'école) et les barèmes de salaire.

Pour certains commentateurs, ces avantages sont trompeurs. Les établissements publics disposaient déjà d'un haut niveau d'autonomie, disent-ils, et l'autonomie pour déterminer les programmes scolaires n'est qu'une illusion, car tous les établissements sont tenus de préparer les mêmes examens nationaux (les GCSE à 16 ans et les A-level à 18 ans ou leurs équivalents techniques et professionnels). Les vraies priorités du gouvernement, à leur avis, sont la réduction des pouvoirs des LA et la déréglementation des conditions de service et des barèmes de salaire.

15 Alors, où en sont les autorités locales? Cela dépend de quelle autorité locale on parle. À York, par exemple, les établissements considèrent que le soutien fourni par l'autorité 
locale est exemplaire et, jusqu'à maintenant, aucun établissement public secondaire n'a demandé à changer de statut. À Darlington, en revanche, presque tous les établissements secondaires se sont déjà convertis, si bien que le rôle de l'autorité locale se ramène plus ou moins à celui d'un prestataire de services en concurrence avec d'autres prestataires commerciaux.

Certains échecs ont été retentissants: ainsi, plusieurs academies ont été jugées « insuffisantes » par le service d'inspection (l'ofsted), preuve s'il en est que le simple fait de changer de statut légal ne garantit pas le succès. Mais il est trop tôt pour évaluer l'efficacité de la stratégie éducative du gouvernement, même si David Cameron a déclaré le 14 novembre 2011: "Je sais que les écoles libres marchent (...) D'ici septembre 2012, nous en aurons créé quatre-vingts ». Apparemment, six semaines lui avaient suffi pour conclure que les free schools fonctionnaient, et cela sans la moindre analyse fournie par l'ofsted.

Qui peut proposer la création d'une free school? Les parents, les enseignants, les mécènes des academies, les écoles indépendantes, les universités, les actions caritatives, les entreprises (sans but lucratif). Le gouvernement continue à promouvoir l'expansion des free schools et affirme que «la procédure est robuste. » Les projets doivent être « créateurs et innovants».

18 Le 28 janvier 2012, le gouvernement est allé encore plus loin dans sa "révolution » en accordant à Internationella Engelska Skolan (IES) un contrat de dix ans d'une valeur de £21 millions pour gérer Breckland Middle School dans le Suffolk. Or IES est une entreprise suédoise à but lucratif qui dégage déjà $€ 5$ millions de profit de ses activités éducatives. Pour certains, la marchandisation de l'école devient une réalité. Pour d'autres, le « profit » n'est pas en question, car le contrat porte sur un « prix fixe ».

19 Mais IES n'est pas la seule entreprise concernée. Une autre entreprise suédoise, Kunskapsskolan, gère déjà trois academies sans but lucratif. Les deux entreprises aspirent maintenant à gérer des réseaux de cinq à dix free schools pour créer des " économies d'échelle ». Et une entreprise britannique, Wey Education, cherche également à s'implanter. Dans un document financier soumis à la Bourse en décembre 2011, l'entreprise estime que « la déconstruction de la fonction éducative des LA offre un fort potentiel pour un important retour sur investissement pour les investisseurs. »

Quand les prochaines élections britanniques auront lieu, en 2015, on saura si le gouvernement vient de faire sauter la digue qui protégeait l'École des grands groupes privés ou si le contrat accordé à IES n'est qu'un cas isolé. On saura aussi si la révolution des academies et des free schools aura abouti à une amélioration du système éducatif dans son ensemble. Les trois années à venir seront fascinantes.

\section{NOTES}

1. L'Écosse et le Pays de Galles ont des systèmes éducatifs qui leur sont propres. La multiplicité d'établissements scolaires décrite ici est une spécificité anglaise. 
2. Le "privé ", en Angleterre, désigne des écoles indépendantes et payantes dont les frais de scolarité sont très élevés : $€ 14000$ en moyenne par année scolaire en externat et jusqu'à $€ 30000$ par année scolaire en internat.

3. Times Educational Supplement, octobre 2010.

\section{INDEX}

Index géographique : Angleterre, Royaume-Uni

Mots-clés : autonomie, établissement d'enseignement, établissement public, politique éducative, système scolaire, enseignement privé

\section{AUTEUR}

\section{DONALD LILLISTONE}

Donald Lillistone a travaillé dans le système éducatif anglais pendant trente-cinq ans comme enseignant, cadre intermédiaire, proviseur adjoint, puis chef d'établissement jusqu'en 2011. Il a été président de l'Association des chefs d'établissement du diocèse de Middlesbrough. Il a écrit des articles sur l'éducation pour le Times Educational Supplement, et pour Leader, la revue de l' Association of School and College Leaders (ASCL). Depuis 2011, il est Foundation Governor de la St Augustine's School à Middlesbrough. 